\title{
Human Ventricular Modelling and Simulation of Drug Action on Electrophysiology and Contraction
}

\author{
Francesca Margara $^{1}$, Zhinuo J Wang ${ }^{1}$, Alfonso Bueno-Orovio ${ }^{1}$, Blanca Rodriguez ${ }^{1}$ \\ ${ }^{1}$ Department of Computer Science, University of Oxford, Oxford, United Kingdom
}

\begin{abstract}
Drug safety and efficacy assessment remains as one of the biggest challenges in both preclinical and clinical drug development. Cardiac adverse outcomes may emerge even though they did not occur in early stages of drug development. Among them, the prediction of drug action on cardiac contraction and electrophysiology is especially complex. Human in-silico drug trials constitute a powerful methodology for their investigation and can integrate and augment biophysically detailed experimental information.

In this study, we present an integrated modelling and simulation framework for the simultaneous assessment of electrophysiological and contractile effects of drug action in human cardiac function. We analyse both pure potassium and calcium channels blockers, given their prevailing use in clinical practice. Simulation results demonstrate the positive inotropic effect of potassium blockers, with the potential occurrence of contractile abnormalities triggered by repolarisation abnormalities, and the dose-dependent negative inotropic effect of calcium blockers. This study demonstrates the translational and preclinical potential of human-based in-silico drug trials to investigate drug-induced effects on human cardiac electromechanical function.
\end{abstract}

\section{Introduction}

In the last six decades, biophysically detailed information on human cardiac (patho-)physiology has been successfully integrated in computational modelling and simulation frameworks. At the basic science level, this has facilitated and augmented mechanistic investigations of cardiac function and disease [1]. Industrial and regulatory bodies have also shown interest in such tools for the evaluation of the safety and efficacy of new or existing therapeutic interventions [2]. In particular, the prediction of clinical cardiotoxicity has recently benefitted from the use of computational approaches to predict pro-arrhythmia liabilities [3]. However, reliable tools for the assessment of drug-induced effects on cardiac contractility are still lacking.

In this study, we present an integrative investigation for the evaluation of drug safety and efficacy in human electromechanical function, using a human computer model constructed and evaluated with experimental data. In particular, through the simulation of pure potassium and calcium blockers, we show the potential of a combined approach that evaluate simultaneously drug-induced effect on both cellular contractility and electrophysiology.

\section{Methods}

The electromechanical function of human ventricular cardiomyocytes was modelled through the coupling of the human-based (A) ToR-ORd [4] and (B) Land [5] models, which describe (1) human ventricular electrophysiology, (2) excitationcontraction coupling and (3) contractility.

Block of the L-Type calcium $\left(\mathrm{I}_{\mathrm{CaL}}\right)$ and slow delayed rectifier potassium $\left(\mathrm{I}_{\mathrm{Kr}}\right)$ currents was simulated to investigate their effects on cellular electromechanical function. Scaling factors were applied to modulate these channel conductances to incorporate the effect of drugs into the model. $\mathrm{I}_{\mathrm{Kr}}$ conductance was scaled to simulate a 50, 80, 95, and $100 \%$ channel block, while $\mathrm{I}_{\mathrm{CaL}}$ conductance was scaled to simulate a $10,30,50$, and $80 \%$ channel block.

The occurrence of electrophysiological and contractile abnormalities following drug exposure was quantified by evaluating the changes in the action potential (AP), calcium transient (CaT), and active tension waveforms, using the following biomarkers: AP duration (APD), active tension amplitude, and the development of both early afterdepolarisations (EADs) and aftercontractions. Occurrences of EADs and aftercontractions were identified as a positive change in the slope of the AP repolarisation phase and of the contractility relaxation transient, before the following stimulusinduced AP and contraction, respectively.

Simulations were conducted using MatLab (Mathworks Inc. Natwick, MA, USA) using the ordinary differential equation solver ode15s. A stimulus current of $-53 \mu \mathrm{A} / \mu F$ with $1 \mathrm{~ms}$ duration was applied and steady state was reached at $1 \mathrm{~Hz}$ pacing before drugs were applied. After an additional 200 beats of simulation at $1 \mathrm{~Hz}$ pacing frequency (unless otherwise stated) the final beats were saved and inspected for the evaluation of electrophysiological and contractile effects as the result of drug exposure. 

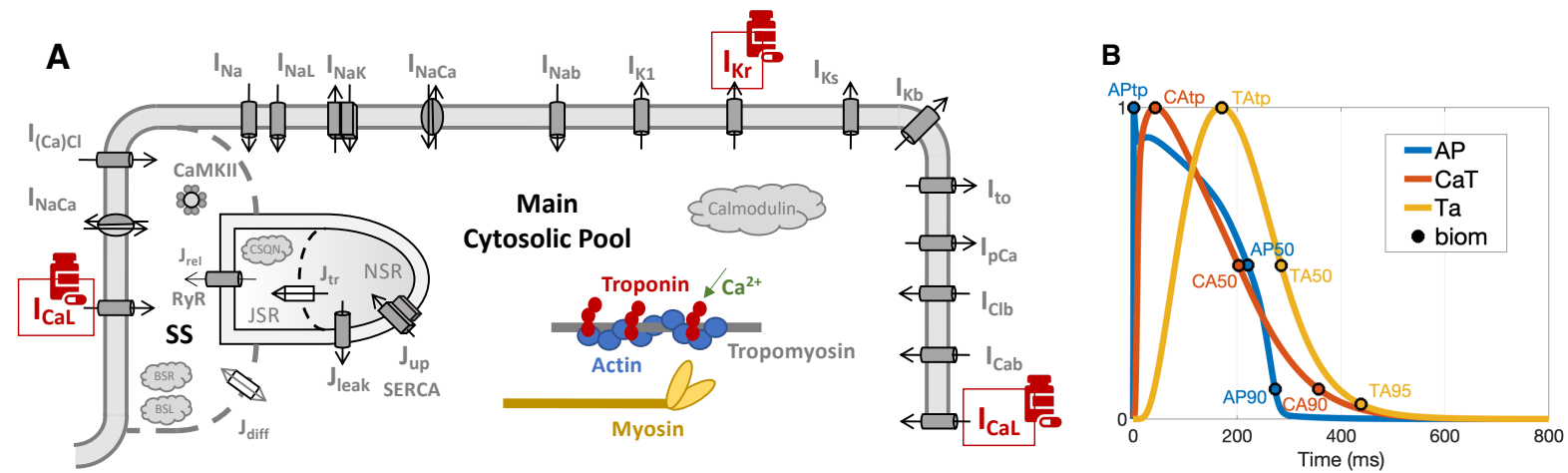

Figure 1. A. Human ventricular model of cellular electromechanical function. Red boxes illustrate the current blocks applied to simulate drug effects. B. Simultaneously simulated action potential (AP), calcium transient $(\mathrm{CaT})$, and active tension (Ta). Circles indicate kinetics biomarkers: time to peak (APtp, CAtp, TAtp), time to $50 \%$ depolarisation/relaxation (APD50, CA50, TA50), and time to 90/95\% repolarisation/relaxation (APD90, CA90, TA95), for the three waveforms.

\section{Results}

The electromechanical model obtained from the coupling of the ToR-ORd and Land models was previously calibrated and evaluated with experimental data, providing a detailed humanbased description of ventricular electrophysiology, excitation-contraction coupling, and contractile cell machinery. The simultaneously simulated AP, CaT, and active tension are reported in Figure 1 along with the diagram of the biophysical model. All AP, $\mathrm{CaT}$, and active tension biomarkers fall within experimental ranges as reported for human samples [6-10].

$I_{K r}$ blockers. Different degrees of $I_{\mathrm{Kr}}$ block were simulated, from $50 \%$ block to full current block, at 1 and $0.25 \mathrm{~Hz}$ pacing. Results in Figure 2A show that, at $1 \mathrm{~Hz}$ pacing, mild degrees of current block lead to a positive inotropic effect mediated by an increase of intracellular calcium due to the AP prolongation induced by the simulated drug. For higher degrees of current block, the significant APD prolongation allows for re-activation of the $\mathrm{I}_{\mathrm{CaL}}$ current. This triggers EADs formation at the lower pacing frequency tested $(0.25 \mathrm{~Hz})$, as show in Figure 2B. In agreement with experimental findings, drugs known to block the $\mathrm{I}_{\mathrm{Kr}}$ current, such as the class III antiarrhythmic drug Dofetilide, have been reported to induce electrical repolarisation abnormalities in the form of EADs [11], as well as contractile abnormalities in the form of aftercontractions [12].

$I_{\text {CaL }}$ blockers. A progressive block of the $\mathrm{I}_{\mathrm{CaL}}$ current determines a block-dependent reduction in cellular inotropy, as illustrated in Figure 3, showing the reduction in peak systolic active tension relative to control. Given the tight relationship between cytosolic calcium levels and force production in ventricular cardiomyocytes, a $50 \% \mathrm{I}_{\mathrm{CaL}}$ block is already sufficient to suppress almost entirely cell contraction.

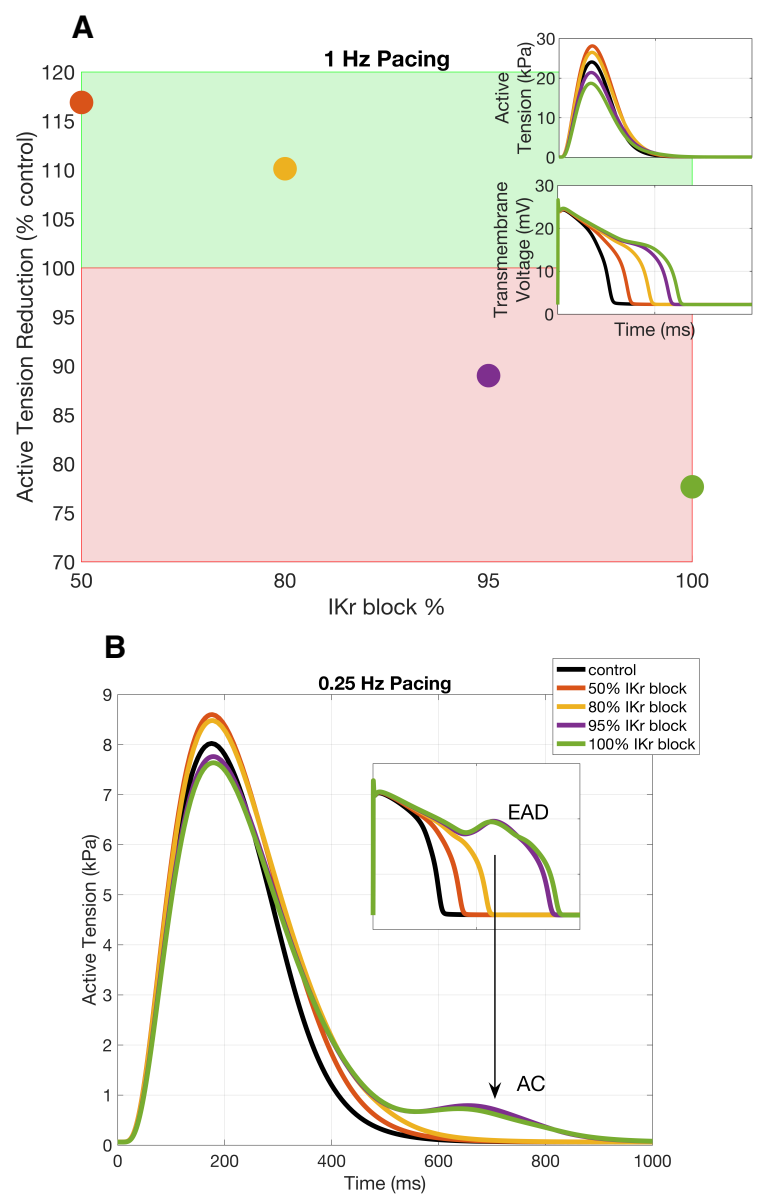

Figure 2. In-silico evaluation of $\mathrm{I}_{\mathrm{Kr}}$ current block on human electromechanical function, simulated at 1 $\mathrm{Hz}(\mathrm{A})$ and $0.25 \mathrm{~Hz}(\mathrm{~B})$. A. Active tension reduction relative to control for different degrees of $\mathrm{I}_{\mathrm{Kr}}$ block. Insets illustrate drug-induced changes in active tension and AP waveforms. B. Aftercontraction (AC) formation triggered by early afterdepolarisation (EAD, inset) at different $\mathrm{I}_{\mathrm{Kr}}$ block levels. Control waveforms reported in black. 
Simultaneously, we were able to evaluate the effects of varying degrees of L-type calcium channel block on the AP waveform. We report a magnitudedependent APD shortening of 4, 13, 25, and $50 \mathrm{~ms}$ for the $10,30,50$, and $80 \%$ of $\mathrm{I}_{\mathrm{CaL}}$ block, respectively. The dose-dependent negative inotropic effects of $\mathrm{I}_{\mathrm{CaL}}$ blockers have been experimentally reported before, showing for example a concentration-dependent decrease in sarcomere shortening under Verapamil exposure [12].

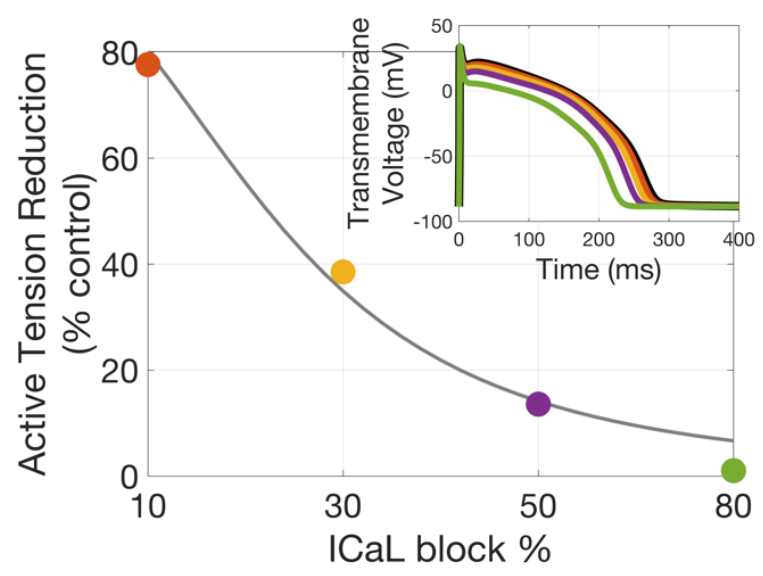

Figure 3. In-silico evaluation of $\mathrm{I}_{\mathrm{CaL}}$ current block on human electromechanical function. Active tension reduction following drug exposure is reported relative to control. Inset: drug effects on AP waveform (control in black).

\section{Discussion}

In this study, we present the development and use of a human-relevant approach for the investigation of both electrophysiological and contractile effects of drug action in human cardiac function. This is achieved by integrating state-of-the-art human models of cellular electrophysiology and contractility, whose coupling provides extended capabilities to concurrently investigate and replicate action potential, calcium dynamics and force generation features, in line with experimental data.

We tested the effects of pure blocks of $\mathrm{I}_{\mathrm{Kr}}$ and $\mathrm{I}_{\mathrm{CaL}}$ currents, demonstrating the positive inotropic effect of mild potassium blocks, and the dose-dependent negative inotropic effect of calcium blockers. Strong potassium current blocks are shown to be capable of inducing electrophysiologically-driven contractile abnormalities, as experimentally reported.

Potassium and calcium blockers, which belong to class III and class IV of the classification of antiarrhythmic drugs, respectively, are both known to present several challenges in their clinical use. Potassium blockers have the potential of prolonging the QT interval and may induce arrhythmias, while calcium blockers can compromise systolic cardiac contraction and worsen certain cardiac diseases (e.g. heart failure). However, little is yet known about the complex interactions between the electrical and mechanical cardiac substrates under drug exposure.

The computational approach presented in this study, combined with experimental data, represents a step forward to better understand the mechanisms behind drug safety and efficacy.

\section{Conclusion}

This study presents a novel human-relevant modelling and simulation framework for the integrative investigation of human ventricular electrophysiological and contractile function, in health, disease, and following therapeutic intervention. Altogether, our findings further support the translational potential of human-based in-silico drug trials for investigations on drug safety and efficacy, extending previous efforts to the evaluation of human cardiac contractile function.

\section{Acknowledgments}

This work was funded by the Personalised InSilico Cardiology (PIC) project, European Union's Horizon 2020 research and innovation programme under the Marie Sklodowska-Curie grant agreement 764738, a Wellcome Trust Fellowship in Basic Biomedical Sciences to B.R. (214290/Z/18/Z), a British Heart Foundation (BHF) Intermediate Basic Science Fellowship to A.B. (FS/17/22/32644), the CompBioMed 1 and 2 Centre of Excellence in Computational Biomedicine (European Commission Horizon 2020 research and innovation programme, grant agreements No. 675451 and No. 823712), an NC3Rs Infrastructure for Impact Award (NC/P001076/1), the TransQST project (Innovative Medicines Initiative 2 Joint Undertaking under grant agreement No 116030, receiving support from the European Union's Horizon 2020 research and innovation programme and EFPIA), and the Oxford BHF Centre of Research Excellence (RE/13/1/30181).

\section{References}

[1] J. Corral-Acero, F. Margara, M. Marciniak, et al. "The 'Digital Twin' to enable the vision of precision cardiology”, Eur. Heart J., 2020 doi: 10.1093/eurheartj/ehaa159.

[2] T. M. Morrison, P. Pathmanathan, M. Adwan, et al. "Advancing Regulatory Science With Computational Modeling for Medical Devices at the FDA's Office of Science and Engineering Laboratories", Front. Med., vol. 5, 241, 2018.

[3] E. Passini, O.J. Britton, H.R. Lu, et al. "Human In Silico Drug Trials Demonstrate Higher Accuracy than Animal Models in Predicting Clinical ProArrhythmic Cardiotoxicity”. Front. Physiol., vol. 8, 668, 2017. 
[4] J. Tomek, A. Bueno-Orovio, E. Passini, et al. "Development, calibration, and validation of a novel human ventricular myocyte model in health, disease, and drug block". eLife, vol. 8, e48890, 2019.

[5] S. Land, S.J. Park-Holohan, N.P. Smith, et al. "A model of cardiac contraction based on novel measurements of tension development in human cardiomyocytes". J. Mol. Cardiol., vol. 106, pp. 6883, 2017.

[6] B. Pieske, M. Sütterlin, S. Schmidt-Schweda, et al. "Diminished post-rest potentiation of contractile force in human dilated cardiomyopathy. Functional evidence for alterations in intracellular $\mathrm{Ca} 2+$ handling". J. Clin. Invest., vol. 98, pp. 764-776, 1996.

[7] L.A. Mulieri, G. Hasenfuss, B. Leavitt, et al. "Altered myocardial force- frequency relation in human heart failure". Circulation, vol. 85, pp. 1743-1750, 1992.

[8] E. Rossman "Abnormal frequency-dependent responses represent the pathophysiologic signature of contractile failure in human myocardium". J. Mol. Cardiol., vol. 36, pp. 33-42, 2004.

[9] R. Coppini, C. Ferrantini, L. Yao, et al. "Late Sodium Current Inhibition Reverses Electromechanical Dysfunction in Human Hypertrophic Cardiomyopathy". Circulation, vol. 127, pp. 575584, 2013.
[10] O.J. Britton, A. Bueno-Orovio, L. Virág, et al. “The Electrogenic $\mathrm{Na}+/ \mathrm{K}+$ Pump Is a Key Determinant of Repolarization Abnormality Susceptibility in Human Ventricular Cardiomyocytes: A Population-Based Simulation Study". Front. Physiol., vol. 8, 278, 2017.

[11] D. Guo, Q. Liu, T. Liu, et al. "Electrophysiological Properties of HBI-3000: A New Antiarrhythmic Agent With Multiple-channel Blocking Properties in Human Ventricular Myocytes". J. Cardiovasc. Pharm. T., vol. 57, pp. 79-85, 2011.

[12] N. Nguyen, W. Nguyen, B. Nguyenton, et al. "Adult Human Primary Cardiomyocyte-Based Model for the Simultaneous Prediction of Drug- Induced Inotropic and Pro-arrhythmia Risk". Front. Physiol., vol. 8, 1073, 2017.

Address for correspondence:

Francesca Margara

Department of Computer Science, Wolfson Building, Parks Road, Oxford, OX1 3QD (UK).

francesca.margara@keble.ox.ac.uk 